\title{
Restoration of microRNA-29c in type I endometrioid cancer reduced endometrial cancer cell growth
}

\author{
MICHELLE VAN SINDEREN ${ }^{1,2^{*}}$, MEAGHAN GRIFFITHS ${ }^{1-3^{*}}$, \\ ELLEN MENKHORST ${ }^{1,2,4^{*}}$, KEITH NIVEN ${ }^{5}$ and EVDOKIA DIMITRIADIS ${ }^{1-4}$
}

\author{
${ }^{1}$ Embryo Implantation Laboratory, Centre for Reproductive Health, Hudson Institute of Medical Research, \\ Clayton, Victoria 3186; Departments of ${ }^{2}$ Molecular and Translational Medicine and ${ }^{3}$ Anatomy and Developmental Biology, \\ Monash University, Clayton, Victoria 3800; ${ }^{4}$ Department of Obstetrics and Gynaecology, \\ The University of Melbourne, The Royal Women's Hospital, Parkville, Victoria 3010; \\ ${ }^{5}$ FlowCore, Technology Research Platforms, Monash University, Clayton, Victoria 3800, Australia
}

Received August 16, 2018; Accepted June 4, 2019

DOI: $10.3892 / 01.2019 .10588$

\begin{abstract}
Endometrial cancer is the most common gynaecological cancer worldwide, and the prognosis of patients with advanced disease remains poor. MicroRNAs (miRs) are dysregulated in endometrial cancer. miRs-29-a, -b and -c expression levels are downregulated in endometrial cancer; however, a specific role for miR-29c and its target genes remain to be elucidated. The aim of the present study was to determine the functional effect of restoring miR-29c expression in endometrial cancer cell lines and to identify miR-29c targets involved in cancer progression. miR-29c expression in human endometrial tumour grades 1-3 and benign tissue as well as in the endometrial cancer cell lines Ishikawa, HEC1A and AN3CA were analysed using reverse transcriptase-quantitative PCR (RT-qPCR). The cell lines were transfected with miR-29c mimic, miR-29c inhibitor or scrambled control. xCELLigence real-time cell monitoring analysed proliferation and migration, and flow cytometry was used to analyse apoptosis and cell cycle. The expression of miR-29c target genes in transfected cell lines was analysed using RT-qPCR. miR-29c was downregulated in grade 1-3 endometrial cancer samples compared with benign endometrium. miR-29c was reduced in Ishikawa and AN3CA cells, but not in HEC1A cell lines compared with non-cancerous primary human endometrial epithelial cells. Overexpression of miR-29c variably reduced proliferation, increased apoptosis and reduced the expression
\end{abstract}

Correspondence to: Professor Evdokia Dimitriadis, Department of Obstetrics and Gynaecology, The University of Melbourne, The Royal Women's Hospital, 20 Flemington Road, Parkville, Victoria 3010, Australia

E-mail: eva.dimitriadis@unimelb.edu.au

*Contributed equally

Key words: endometrial cancer, uterus, microRNA, proliferation, target genes, Ishikawa, AN3CA, HEC1A levels of miR-29c target genes, including cell division cycle 42 , HMG-box transcription factor 1 , integrin subunit $\beta 1$, MCL1 apoptosis regulator BCL2 family member, MDM2 proto-oncogene, serum/glucocorticoid regulated kinase 1, sirtuin 1 and vascular endothelial growth factor $\mathrm{A}$, across the three cell lines investigated. Inhibition of miR-29c in HEC1A cells increased proliferation and collagen type IV $\alpha 1$ chain expression. The re-introduction of miR-29c to endometrial cancer cell lines reduced proliferation, increased apoptosis and reduced miR-29c target gene expression in vitro. The present results suggested that miR-29c may be a potential therapeutic target for endometrial cancer.

\section{Introduction}

Endometrial cancer (EC) is the most common female gynaecological cancer worldwide with 280,000 new cases per year (1). Alarmingly, the incidence of EC is on the rise, particularly in younger women of reproductive age (2), and this is believed to be caused by increasing rates of societal obesity predominantly in developed countries (3). There are currently no effective early diagnostic screening tests, and advanced or recurrent EC presents a poor prognosis. EC is broadly categorized into two major types, and $80-90 \%$ of EC are classified as type I (4). Type I EC are associated with unopposed oestrogen and endometrial hyperplasia and, depending on the extent of solid tumour growth, Type I EC can be further divided into grades based on differentiation status: i) Grade 1 (G1), well differentiated; ii) grade 2 (G2), moderately differentiated; and iii) grade 3 (G3), poorly differentiated (5). Type II $\mathrm{EC}$ occurs less frequently, but is more aggressive than type I, it is not associated with oestrogen stimulation and arises from a state of atrophy (5). However, individual EC tumours can have diverse molecular and genomic profiles; therefore, treatments based on histological classification alone may not be effective (6). This limitation highlights the need for molecular characterization, which may facilitate the use of personalised therapies (7).

MicroRNAs (miRs) are a class of small (18-22 nucleotides), highly conserved, non-coding RNAs that regulate 
gene expression post-transcriptionally (8). The mature form of miRs can act by destabilising mRNAs or by repressing protein translation, leading to a downregulation of the target protein products, influencing their biological functions (8). miR expression is tightly coordinated and each miR has the ability to act on numerous target genes, regulating some of the most basic cellular processes such as proliferation, apoptosis and metabolism (8).

The miR-29 family includes miR-29-a, -b and -c, which share an identical seed sequence and mature sequences with only minor nucleotide differences, and thus share many predicted targets (9). Members of the miR-29 family are implicated in targeting genes encoding extracellular matrix proteins, transcription factors, cell cycle proteins, factors involved in cytokine signalling, phosphatases and proteins with roles in many other biological processes (9). miR-29-a, -b and -c have all been shown to be downregulated in EC (10-14); however, the specific role for miR-29c and its targets in EC growth and development are unknown.

miR-29c is located and transcribed from chromosome 1q32.2. miR-29c is an established tumour suppressor in many cancer types including lung, gastric, pancreatic and bladder cancer (15). miR-29c expression is elevated in early and mid-secretory phase endometrial epithelium of women with primary infertility (16). Elevated endometrial miR-29c impairs endometrial epithelial collagen type IV a1 (COL4A1) production, likely impairing the adhesive capacity of the luminal epithelium and contributing to implantation failure and infertility (16). miR-29c also contributes to endometriosis-induced infertility by affecting endometriosis cell proliferation, invasion and apoptosis (17).

The aims of the present study were to define miR-29c expression in endometrioid (type I) EC, to investigate the functional effects of re-introducing miR-29c expression in EC cell lines and to identify miR-29c targets involved in cancer progression in order to pinpoint future potential therapeutic targets.

\section{Materials and methods}

Patient samples. The present study was approved by the Monash Health Human Research and Ethics Committee (approval no. 06014C) and the Victorian Cancer Biobank (Melbourne, Australia; project no. 13018). Informed consent was obtained from each participant at the time of sample collection $(n=35)$.

EC samples. EC samples were collected as follows from female patients with the following characteristics. The Victorian Cancer Biobank provided RNA from type I EC ( $n=10$ samples/grade) or benign post-menopausal endometrium $(n=5)$ whole tissue. There was no difference in patient age between the three EC grades (age of patients with G1, $53.4 \pm 3.5$ years; age of patients with $\mathrm{G} 2,63.8 \pm 4.2$ years; age of patients with $\mathrm{G} 3,66.1 \pm 4.3$ years), whereas age information was not available for patients with benign endometrium. The patients did not receive chemotherapy, radiotherapy or other biological targeted therapy prior to surgery. Samples were collected by the Victoria Cancer Biobank between 2007 and 2014.
EC cell line culture. G1-derived Ishikawa cells were provided by Dr Nishida (Tsukuba University, Tochigi, Japan) and cultured in DMEM/F12 medium (Thermo Fisher Scientific, Inc.) supplemented with $10 \%$ foetal calf serum (FCS; Invitrogen; Thermo Fisher Scientific, Inc.). G2-derived HEC1A cells [authenticated by the Monash Health Translation Precinct (MHTP) Medical Genomics Facility] were purchased from the American Type Culture Collection (ATCC) and cultured in McCoy's medium (Thermo Fisher Scientific, Inc.) supplemented with $10 \%$ FCS. G3-derived AN3CA cells (authenticated by the MHTP Medical Genomics Facility) were purchased from the ATCC and cultured in DMEM/F12 medium supplemented with $10 \%$ FCS. All cells were cultured in a humidified incubator maintained at $37^{\circ} \mathrm{C}$ in an atmosphere with $5 \% \mathrm{CO}_{2}$.

Primary epithelial cell isolation and culture. Endometrial biopsies were collected at curettage from women with regular menstrual cycles at the proliferative stage (days 7-13) of the menstrual cycle. The women had no steroid treatment for $\geq 2$ months prior to tissue collection. The biopsies were examined by an experienced gynaecological pathologist to confirm the stage and absence of endometrial dysfunction. Human endometrial epithelial cells (HEEC) were prepared as previously described (18). Briefly, endometrial tissue was digested with collagenase and the suspension was filtered through nylon mesh to collect endometrial epithelial glands. The epithelial glands were collected and resuspended in DMEM/F12 supplemented with $10 \%$ FCS and $1 \%$ Penicillin-Streptomycin antibiotic-antimycotic solution (Gibco; Thermo Fisher Scientific, Inc.) before being serially replated for three times in plastic culture dishes for $30 \mathrm{~min}$ in a humidified incubator with $5 \% \mathrm{CO}_{2}$ at $37^{\circ} \mathrm{C}$ to allow adherence of contaminating stromal cells. Non-adherent cells and glands were transferred to 96 or 48 -well plates and epithelial cells were allowed to grow out from glandular structures for $48 \mathrm{~h}$ in a humidified incubator with $5 \% \mathrm{CO}_{2}$ at $37^{\circ} \mathrm{C}$. A purity of $>95 \% \mathrm{HEEC}$ as previously described (18) was necessary for the cells to be used experimentally. Confluent HEEC were cultured in serum-free DMEM/F12 containing 1\% Penicillin-Streptomycin solution for $16 \mathrm{~h}$ prior to RNA extraction.

miR-29c mimic and miR-29c inhibitor transfection. Ishikawa, HEC1A or AN3CA cells were transfected according to the manufacturer's protocol, as previously described (19) using Lipofectamine ${ }^{\circledR}$ RNAiMAX (Applied Biosystems; Thermo Fisher Scientific, Inc.) and miR-29c mimic (25 nM; hsa-miR-29c-3p; seed sequence: 5'-UCUCUU ACACAGGCUGACCGAUUUCUCCUGGUGUUCAGAGUC UGUUUUUGUCUAGCACCAUUUGAAAUCGGUUAU GAUGUAGGGGGA-3'; cat. no. MC10518; Thermo Fisher Scientific, Inc), miR-29c inhibitor (25 nM; hsa-miR-29c-3p; seed sequence: 5'-AUCUCUUACACAGGCUGACCGAUU UCUCCUGGUGUUCAGAGUCUGUUUUUGUCUAGC ACCAUUUGAAAUCGGUUAUGAUGUAGGGGGA-3'; cat. no. MH10518; Thermo Fisher Scientific, Inc.) for $72 \mathrm{~h}$ in a humidified incubator with $5 \% \mathrm{CO}_{2}$ at $37^{\circ} \mathrm{C}$. A scrambled miR sequence (25 nM; cat. no. 4464058; Thermo Fisher Scientific, Inc.) was used as a control. Transfection efficiency was determined using reverse transcription-quantitative PCR 
Table I. microRNA-29c target gene primer sequences.

\begin{tabular}{|c|c|c|}
\hline Gene & Gene name & Sequence $\left(5^{\prime} \rightarrow 3^{\prime}\right)$ \\
\hline CAV1 & Caveolin-1 & $\begin{array}{l}\text { F: CAGGGACATCTCTACACC } \\
\text { R: TCAAAGTCAATCTTGACCAC }\end{array}$ \\
\hline CDC42 & Cell division cycle 42 & $\begin{array}{l}\text { F: GAACAAACAGAAGCCTATCAC } \\
\text { R: TTTAGGCCTTTCTGTGTAAG }\end{array}$ \\
\hline COL4A1 & Collagen type IV $\alpha 1$ & $\begin{array}{l}\text { F: AAAGGGAGATCAAGGGATAG } \\
\text { R: TCACCTTTTTCTCCAGGTAG }\end{array}$ \\
\hline FBN1 & Fibrillin 1 & $\begin{array}{l}\text { F: AAAGATCTTGATGAGTGTGC } \\
\text { R: GTATGGTGTTGGGTAAATCC }\end{array}$ \\
\hline HBP1 & HMG-box transcription factor 1 & $\begin{array}{l}\text { F: GAATGCCTTCATGCTTTTTG } \\
\text { R: CACACTTATGGCTCTGTTATC }\end{array}$ \\
\hline ITGB1 & Integrin $\beta 1$ & $\begin{array}{l}\text { F: ATTCССТTTCСТCAGAAGTC } \\
\text { R: TTTTCTTCCATTTTCCССTG }\end{array}$ \\
\hline MCL1 & MCL1 apoptosis regulator, BCL2 family member & $\begin{array}{l}\text { F: TAGTTAAACAAAGAGGCTGG } \\
\text { R: ATAAACTGGTTTTGGTGGTG }\end{array}$ \\
\hline MDM2 & MDM2 proto-oncogene & $\begin{array}{l}\text { F: CAGCAGGAATCATCGGACTCA } \\
\text { R: ACACAGAGCCAGGCTTTCAT }\end{array}$ \\
\hline MMP2 & Matrix metalloproteinase 2 & $\begin{array}{l}\text { F: GTGATCTTGACCAGAATACC } \\
\text { R: GCCAATGATCCTGTATGTG }\end{array}$ \\
\hline NUMB & NUMB endocytic adaptor protein & $\begin{array}{l}\text { F: AGGCTCTTTCCGACCTTTTC } \\
\text { R: GCTGAAGGCATTGGTGATCT }\end{array}$ \\
\hline SGK1 & Serum/glucocorticoid regulated kinase 1 & $\begin{array}{l}\text { F: AGACTACATTAATGGTGGAGAG } \\
\text { R: ATTTCAGCAGCATAGAAACG }\end{array}$ \\
\hline SIRT1 & Sirtuin 1 & $\begin{array}{l}\text { F: AAGGAAAACTACTTCGCAAC } \\
\text { R: GGAACCATGACACACTGAATTATC }\end{array}$ \\
\hline VEGFA & Vascular endothelial growth factor A & $\begin{array}{l}\text { F: AATGTGAATGCAGACCAAAG } \\
\text { R: GACTTATACCGGGATTTCTTG }\end{array}$ \\
\hline $18 \mathrm{~S}$ & $18 \mathrm{~S}$ ribosomal RNA & $\begin{array}{l}\text { F: GTAACCCGTTGAACCCCATTC } \\
\text { R: GCCTCACTAAACCATCCAATCG }\end{array}$ \\
\hline
\end{tabular}

F, forward; R, reverse.

(RT-qPCR). Further experiments, including proliferation assay, flow cytometry and RT-qPCR, were performed $72 \mathrm{~h}$ after initial transfection.

$x$ CELLigence real time proliferation assay. Experiments were carried out using the real-time cell analyser (RTCA) DP xCELLigence instrument (ACEA Biosciences; Agilent Technologies $\mathrm{GmbH}$ ), as previously described (20). The $x$ CELLigence instrument was kept in a humidified incubator maintained at $37^{\circ} \mathrm{C}$ with $5 \% \mathrm{CO}_{2}$. Cells were transfected with miR-29c mimic, miR-29c inhibitor or control for $72 \mathrm{~h}$, and seeded in E-plate 96 (ACEA Biosciences; Agilent Technologies $\mathrm{GmbH}$ ) at $\sim 10,000$ cells/well in media (DMEM/F12 for Ishikawa and AN3CA, McCoy for HEC1A) supplemented with $5 \%$ FCS. The plates were monitored every $15 \mathrm{~min}$ for a total of $72 \mathrm{~h}$. Data were analysed using RTCA software v1.2, supplied with the instrument (ACEA Biosciences; Agilent Technologies $\mathrm{GmbH}$ ) and exported for statistical analysis.

Flow cytometry and cell cycle analysis. Ishikawa, HEC1A or AN3CA cells were serum-starved for $24 \mathrm{~h}$ to synchronize populations into $\mathrm{G}_{0}$, before cells were transfected with miR-29c mimic, inhibitor or scrambled miR sequence (as described above). Cells were harvested after $72 \mathrm{~h}$, fixed in $70 \%$ ethanol and stained with FxCycle propidium iodide (PI)/RNase staining solution (Molecular Probes; Thermo Fisher Scientific, Inc.) and analysed on a BDFACSCanto II flow cytometer. Cell cycle, apoptosis analysis and model fitting were performed with FlowJo (Version X, FlowJo LLC, Ashland, OR, USA) (19).

$R N A$ preparation and $R T-q P C R$. RNA was extracted from cultured cells or human epithelial endometrial biopsies using Tri Reagent (Sigma-Aldrich; Merck KGaA) according to the manufacturer's protocol. Genomic DNA was digested using the DNAfree kit (Ambion; Thermo Fisher Scientific, Inc.) according to the manufacturer's protocol. RNA samples concentration, yield and purity were analysed using a spectrophotometer (Nanodrop Technologies; Thermo Fisher Scientific, Inc.) at an absorbance ratio of 260/280 nm.

RNA was reverse transcribed using the TaqMan reverse transcription kit (Applied Biosystems; Thermo Fisher 

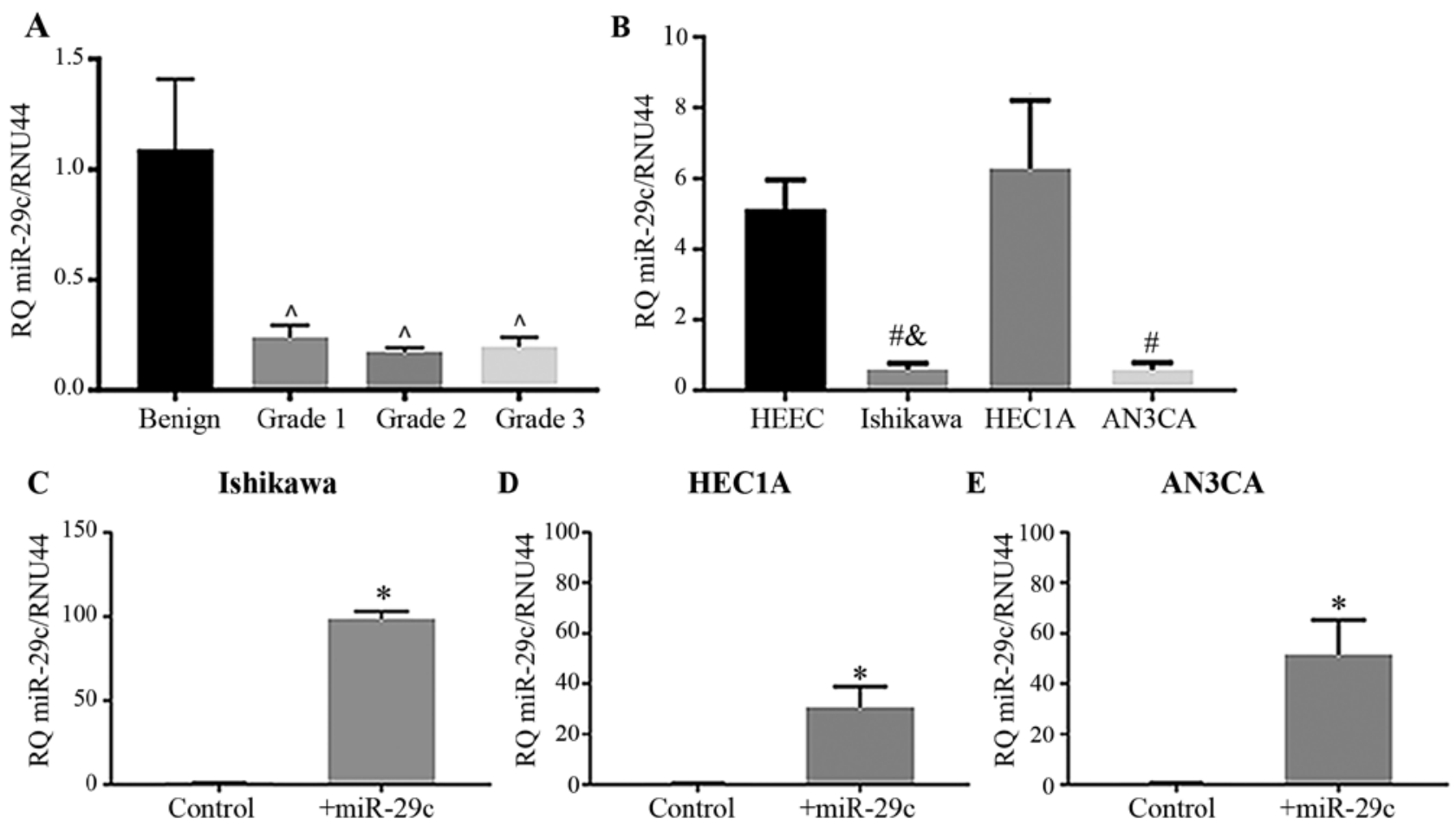

Figure 1. miR-29c expression levels in endometrial cancer. (A) miR-29c expression in human endometrial cancer at grades 1-3 compared with benign biopsies. $\mathrm{n}=10$ in each group. (B) miR-29c expression in human endometrial cancer cell lines at grades 1-3 (n=3-9) compared with that in normal proliferative phase endometrium $(\mathrm{n}=4)$. Ishikawa miR-29c expression was significantly lower compared with HEEC and HEC1A. AN3CA miR-29c expression was significantly lower compared to HEEC. (C-E) Reverse transcription-quantitative PCR validation of the overexpression of miR-29c in transfected (C) Ishikawa, (D) HEC1A and (E) AN3CA endometrial cancer cell lines. $\mathrm{n}=4-9$ in each group. Expression level of miR-29c was normalized to RNU44. Data are presented as the mean $\pm \mathrm{SEM}$; "P<0.05 vs. scrambled miR mimic; ${ }^{\wedge} \mathrm{P}<0.05$ vs. benign tissue; ${ }^{*} \mathrm{P}<0.05$ vs. HEEC; ${ }^{\circledR} \mathrm{P}<0.05$ vs. HEC1A. + miR-29c, transfected with miR-29c mimic; miR, microRNA; HEEC, human endometrial epithelial cell line.

Scientific, Inc), and specific TaqMan primer sets for miRs (miR-29c-3p; cat. no. 000587; rnU44, cat. no. 001094; Applied Biosystems; Thermo Fisher Scientific, Inc.) or with a Moloney Murine Leukemia Virus RT system (Life Technologies; Thermo Fisher Scientific, Inc.) and oligo primers (Sigma Aldrich; Merck KGaA) for non-miR genes. qPCR was performed using TaqMan Fast Universal PCR Master mix (Applied Biosystems; Thermo Fisher Scientific, Inc.) or Power SYBR Green master mix (Applied Biosystems; Thermo Fisher Scientific, Inc.) using TaqMan probes (miR-29c-3p; cat. no. 000587; rnU44, cat. no. 001094; Thermo Fisher Scientific, Inc.) or specific primer pairs (Table I) performed on the ABI 7500HT fast block qPCR system (Applied Biosystems; Thermo Fisher Scientific, Inc.) in triplicate (final reaction volume, $10 \mu \mathrm{l}$ ) in 384-well micro optical plates (Applied Biosystems; Thermo Fisher Scientific, Inc.). A template-free negative control in the presence of primers and RNase-free water, and RNase-free water only negative controls were added for each run. The qPCR protocol was as follows: Initial denaturation at $95^{\circ} \mathrm{C}$ for $10 \mathrm{~min}$, followed by 40 cycles of $95^{\circ} \mathrm{C}$ for $15 \mathrm{sec}$ and $60^{\circ} \mathrm{C}$ for $1 \mathrm{~min}$. miR-29c expression levels were normalised against the control RNU44 probes. Gene expression was normalised against 18 S ribosomal RNA (18S). Relative expression levels were calculated using comparative cycle threshold method $\left(2^{-\Delta \Delta C q}\right) 6$ (21) according to the manufacturer's protocol, with $18 \mathrm{~S}$ serving as the endogenous control for normalization.

Predicted and confirmed miR-29c target genes analysed in this study were chosen by bioinformatics analysis using online databases: picTAR (https://pictar.mdc-berlin.de/ established 2007), TarBase (Version 7, http://diana.imis.athena-innovation. gr/DianaTools/index.php?r=tarbase/index) and TargetScan (Version 7.2, http://www.targetscan.org/vert_72/).

The following miR-29c target genes were analysed using RT-qPCR: Caveolin 1 (CAV1), cell division cycle 42 (CDC42), COL41A, fibrillin 1, HMG-box transcription factor 1 (HBP1), integrin subunit $\beta 1$ (ITGB1), NUMB endocytic adaptor protein (NUMB), MCL1 apoptosis regulator BCL2 family member (MCL1), MDM2 proto-oncogene (MDM2), serum/glucocorticoid regulated kinase 1 (SGK1), sirtuin 1 (SIRT1) and vascular endothelial growth factor A (VEGFA). Primer sequences are presented in Table I.

Statistical analysis. Statistical analysis was carried out using GraphPad Prism (v8.0.1; GraphPad Software, Inc.) and data were assessed by paired Student's t-test for two groups or one-way ANOVA with Tukey's post-hoc test for multiple groups. Real-time xCELLigence monitoring and flow cytometry was assessed using repeated measures two-way ANOVA, with Sidak's post-hoc test. Data are presented as the mean \pm SEM. A minimum of three repeats was required for statistical analysis to be performed. $\mathrm{P}<0.05$ was considered to indicate a statistically significant difference.

\section{Results}

miR-29c expression is downregulated in human type I endometrioid cancer cell lines. miR-29c expression was 


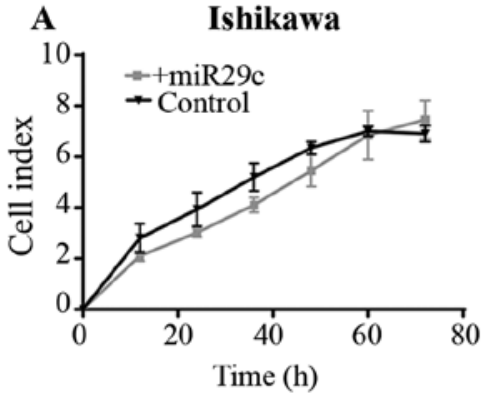

D

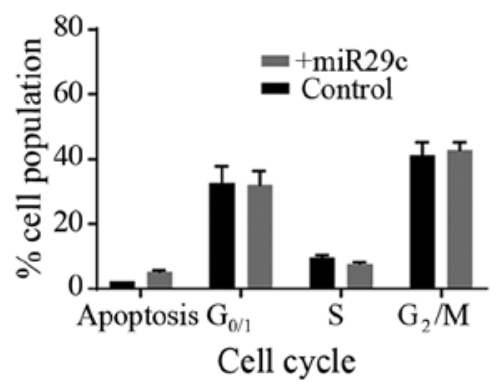

B

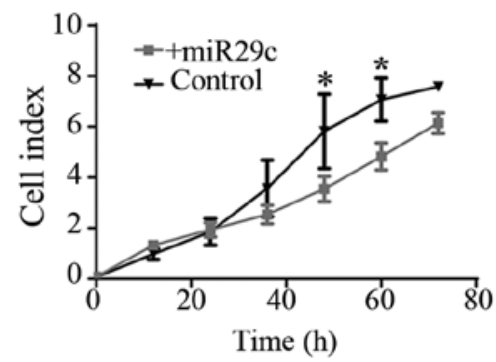

$\mathbf{E}$

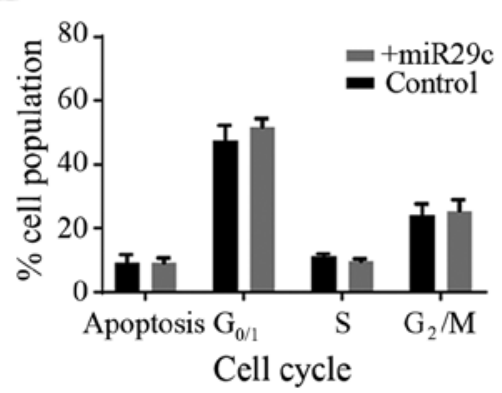

C

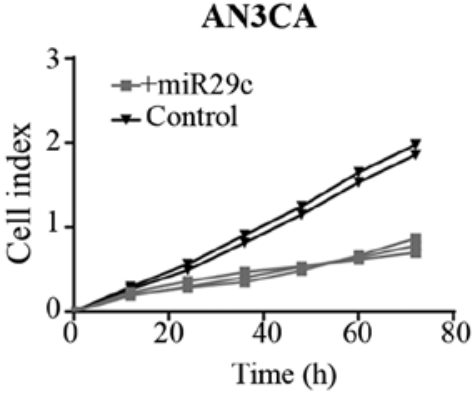

F

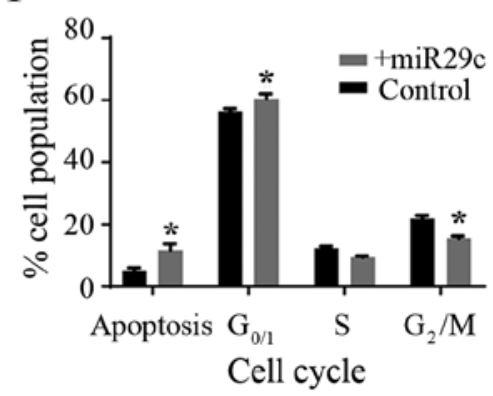

Figure 2. Effects of miR-29c overexpression on proliferation, cell cycle progression and apoptosis. miR-29c overexpression in (A) Ishikawa, (B) HEC1A and (C) AN3CA endometrial cancer cell lines. Reduced proliferation in HEC1A cells was found compared with the control group using xCELLigence real-time cell monitoring. $n=3-4$ in each group. Cell cycle analysis in (D) Ishikawa, (E) HEC1A and (F) AN3CA cells. Increased number of apoptotic cells and in $\mathrm{G}_{0} / 1$ phase, and reduced number of cells in $\mathrm{G}_{2} / \mathrm{M}$ phase were identified by flow cytometry in the AN3CA cell line. $n=4-5$ in each group. Data from three replicates are presented as the mean \pm SEM. ${ }^{*} \mathrm{P}<0.05$ vs. corresponding control.

significantly reduced in human G1, 2 and 3 type I endometrioid cancer biopsies compared with that in the benign endometrium (Fig. 1A; P<0.05). Similarly, miR-29c expression levels were significantly reduced in the EC epithelial cell lines Ishikawa (G1), and AN3CA (G3) compared with that in the primary HEECs (Fig. 1B; $\mathrm{P}<0.05$ ); however, no reduction was seen in HEC1A (G2) cells. Confirmation of miR-29c mimic transfection efficiency in Ishikawa, HEC1A and AN3CA cells showed that miR-29c was significantly upregulated following miR-29c mimic overexpression (Fig. 1C-E; P<0.05).

miR-29c overexpression reduces cell proliferation in G2and G3-derived EC cell lines. To determine the functional role of miR-29c in EC cell proliferation, miR-29c was overexpressed in Ishikawa (G1) HEC1A (G2) and AN3CA (G3) cell lines (Fig. 2A-C). Using the xCELLigence real-time system, miR-29c mimic overexpression was identified to significantly reduce HEC1A proliferation (at 48 and $60 \mathrm{~h}$; $\mathrm{P}<0.05)$ compared with that in the control cells (Fig. 2B). Proliferation was also reduced following miR-29c overexpression in the AN3CA cell line; however, statistical analyses could not be performed, as there were only two control samples (Fig. 2C). There was no effect of miR-29c mimic overexpression on Ishikawa cell proliferation (Fig. 2A).

To determine the effect of miR-29c overexpression on cell cycle and the levels of apoptosis in Ishikawa (G1), HEC1A (G2) and AN3CA (G3) EC cell lines, flow cytometry was performed following PI/RNase staining. There was no effect of miR-29c overexpression in Ishikawa or HEC1A cells compared with the corresponding controls (Fig. 2D and E). However, in AN3CA cells, miR-29c overexpression increased the percentage of apoptotic cells and the rate of cells in $\mathrm{G}_{0} / \mathrm{G}_{1}$ phase, and decreased the percentage of cells in $\mathrm{G}_{2} / \mathrm{M}$ phase (Fig. 2F; P<0.05).

miR-29c overexpression reduces the expression of target genes. miRs act by binding to target sequences in the 3 -untranslated regions of multiple genes. miR-29c regulation of target gene expression is presented in Fig. 3. In the Ishikawa cell line miR-29c overexpression significantly reduced HBP1, ITGB1, MCL1, MDM2 and SGK1 expression compared with the scrambled control. HBP1, ITGB1, MDM2, SIRT1 and VEGFA expression was significantly reduced following miR-29c overexpression in HEC1A cells compared with that in the scrambled control group. CDC42, ITGB1, MCL1 and MDM2 were all significantly reduced in the AN3CA cells following miR-29c overexpression compared with that in the scrambled control group. There was no significant effect on CAV1 or NUMB gene expression in any cell line (data not shown).

miR-29c inhibition in HEClA. Endogenous levels of miR-29c expression in the HEC1A cells were found to be similar to the HEEC control cells and not reduced as in the Ishikawa and AN3CA cancer cell lines (Fig. 1B). Therefore, the effect of inhibiting miR-29c in the HEC1A cells was examined (Fig. 4A). The inhibition of miR-29c expression significantly increased proliferation between 24 and $60 \mathrm{~h}(\mathrm{P}<0.05)$ compared with that in the control group (Fig. 4B). No differences were seen in the cell cycle phases upon the inhibition of miR-29c (Fig. 4C). COL4A1 gene expression was significantly increased following miR-29c inhibition (Fig. 4D). COL4A1 is a known target of miR-29c; overexpression of miR-29c reduces COL4A1 production 

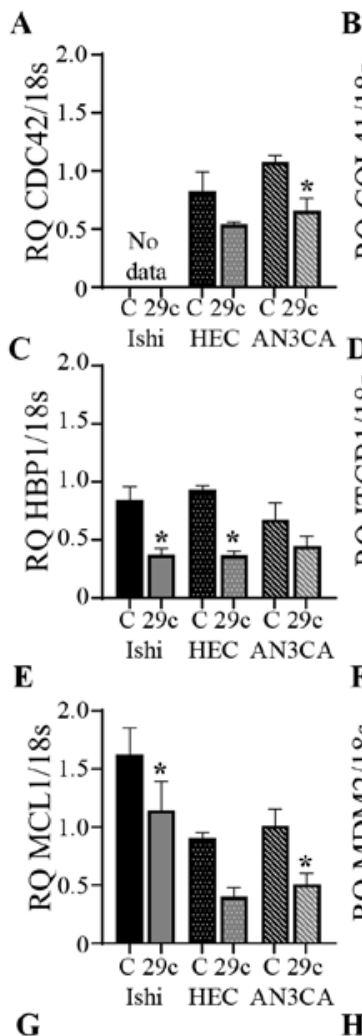

G
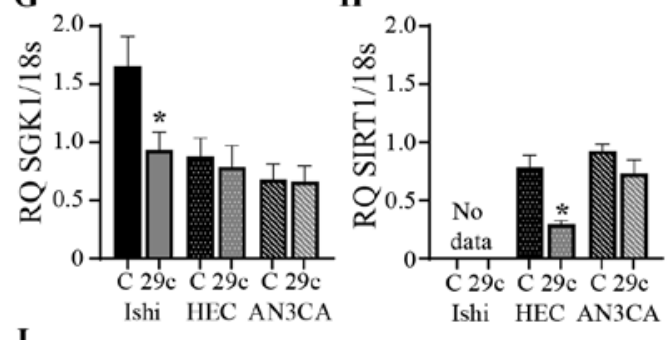

I

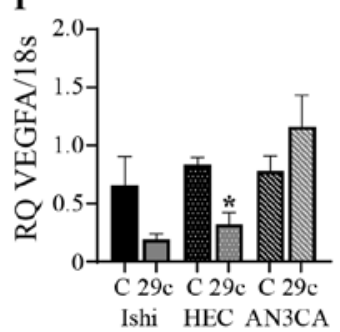

Figure 3. Effects of miR-29c overexpression on the expression levels of its predicted target genes. miR-29c overexpression reduced the expression levels of (A) CDC42, (B) COL4A1, (C) HBP1, (D) ITGB1, (E) MCL1, (F) MDM2, (G) SGK1, (H) SIRT1 and (I) VEGFA in Ishikawa, HEC1A and AN3CA endometrial cancer cell lines, as assessed by reverse transcription-quantitative PCR. Expression levels of all genes were normalized to $18 \mathrm{~s}$. Data are presented as the mean \pm SEM. $n=3-6$ in each group. ${ }^{*} \mathrm{P}<0.05$ vs. corresponding control. 29c, miR-29c overexpression; C, control; Ishi, Ishikawa; HEC, HEC1A; CDC42, cell division cycle 42; COL41 A, collagen type IV $\alpha 1$ chain; HBP1, HMG-box transcription factor 1; ITGB1, integrin subunit $\beta$ 1; MCL1, MCL1 apoptosis regulator, BCL2 family member; MDM2, MDM2 proto-oncogene; SGK1, serum/glucocorticoid regulated kinase 1; SIRT1, sirtuin 1; VEGFA, vascular endothelial growth factor A; 18s, 18S ribosomal RNA; RQ, relative quantification.

in endothelial (22) and endometrial epithelial (16) cells. The expression of the other aforementioned genes identified following bioinformatics analysis were investigated following miR-29c inhibition, but only COL4A1 was significantly altered (data not shown).

\section{Discussion}

To the best of our knowledge, the present study is the first to describe the functional effects and downstream targets of miR-29c in the EC cell lines Ishikawa (G1), HEC1A (G2) and AN3CA (G3). It was identified that miR-29c was downregulated in human G1, G2 and G3 EC tumours compared with benign tissue. Compared with benign primary human endometrial epithelial cells, the EC cell lines Ishikawa (G1) and AN3CA (G3) had reduced endogenous miR-29c levels, whereas HEC1A levels were equivalent to the levels in the benign primary human endometrial epithelial cells. Exogenous miR-29c overexpression reduced proliferation of the HEC1A cell line, increased apoptosis and reduced cell cycle progression in the AN3CA cell line. Conversely, inhibition of miR-29c in HEC1A cells significantly increased cell proliferation and COL4A1 expression. miR-29c target genes, such as ITGB1 and MDM2, which are involved in adhesion, cell survival and migration $(23,24)$, were downregulated following miR-29c overexpression, suggesting that miR-29c may act as a tumour suppressor in EC.

Previous studies showed that miR-29c expression was downregulated in grades 1-3 type I EC tissue compared with that in benign tissue from non-malignant endometrium $(10,14)$. The Ishikawa (G1) and AN3CA (G3) cells also exhibited decreased miR-29c expression compared with that in isolated human endometrial epithelial cells. However, the HEC1A (G2) cell line presented similar endogenous miR-29c levels to the non-cancerous endometrial epithelial control cells. All three of these cell lines are known to harbour different mutational profiles (25), which could potentially be involved in regulating miR-29c expression and may account for differences in functional responses to specific factors, including miR-29c. miR-29c expression can be regulated by different factors influencing its promoter region such as methylation sites (26). Moreover, miR-29c promoter possesses a p53 consensus responsive element (27) which may downregulate its activity. Additionally, miR-29c is regulated by ovarian hormones in uterine leiomyoma smooth muscle cells (28).

miR-29c overexpression reduced cell proliferation in HEC1A cells and cell cycle progression in the AN3CA cell line. Supporting a role of miR-29c in repressing endometrial cell growth, a previous study found that miR-29c overexpression reduced cell growth in the endometriosis cell line CRL-7566, possibly by increasing apoptosis (17). In the present study, the percentage of apoptotic cells was increased only in AN3CA cells. The time-point selected to collect the cells for flow cytometry $(72 \mathrm{~h})$ may be too late to see an effect on apoptosis in HEC1A cells. Moreover, the higher endogenous levels of miR-29c in HEC1A cells may mask the effects of miR-29c overexpression. In the endometriosis cells, apoptosis was suggested to increase following repression of c-Jun (17). c-Jun was not investigated in the present study, but miR-29c reduced expression of the antiapoptotic protein MCL1 in AN3CA cells. It would be of interest to determine whether miR-29c is able to target c-Jun in EC cells, particularly AN3CAs.

It is surprising that no effect on proliferation or cell cycle progression was found in the Ishikawa cell line; however, this may be due to the peculiar mutational profile found in this 

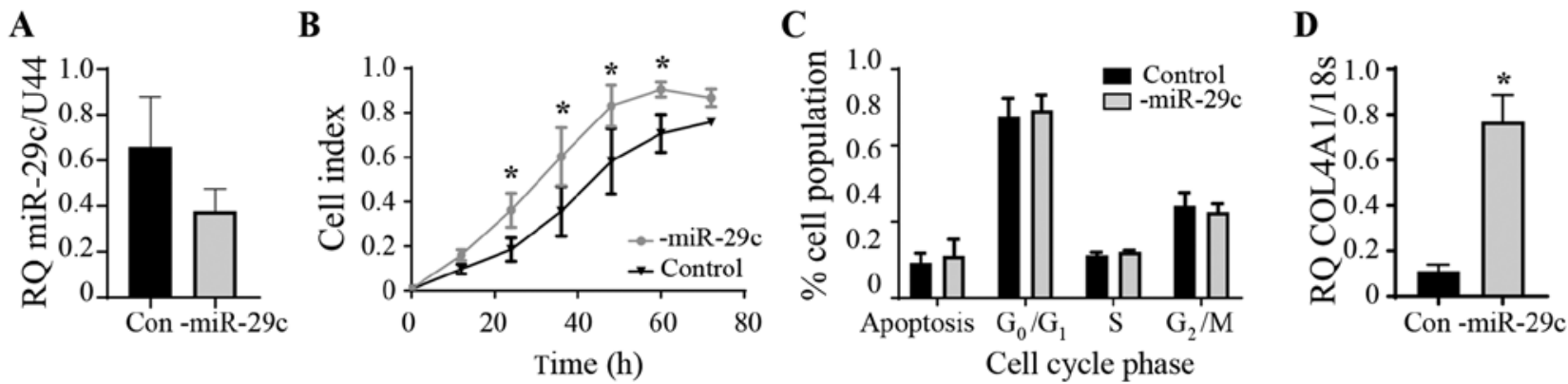

Figure 4. miR-29c inhibition in HEC1A cells. (A) miR-29 expression was not significantly reduced. (B) Cell proliferation increased using xCELLigence real-time cell monitoring. $\mathrm{n}=5$ in each group. (C) There was no effect on apoptosis or cell cycle using flow cytometry. $\mathrm{n}=5$ in each group. (D) Increased COL4A1 mRNA expression. $\mathrm{n}=4$ in each group. Expression level of COL4A1 was normalized to 18s. Expression level of miR-29c was normalized to RNU44. Data are presented as the mean \pm SEM. ${ }^{*} \mathrm{P}<0.05$ compared with the control group. miR, microRNA; Con, control; RQ, relative quantification; COL4A1, collagen type IV $\alpha 1$ chain.

cell line (25). However, miR-29c overexpression repressed the expression of various target genes in the three cell lines investigated. Notably, more detailed studies investigating the effect of miR-29c on apoptosis and cell cycle progression in EC are required.

miR-29c predicted and confirmed target genes involved in cellular growth, adhesion, migration and invasion were examined by qPCR. The present data suggested that these genes are regulated by miR-29c; however, luciferase reporter assays are required to demonstrate direct targeting of these genes by miR-29c. Interestingly, variation in miR-29c target genes was found between the three EC cell lines. This phenomenon is likely due to the variable level of endogenous miR-29c and the specific mutational profiles within each individual cell line (detailed in 25). For example, Ishikawa and AN3CA cells exhibit different mutations in PTEN, leading to the absence of the protein, whereas HEC1A has no mutations and PTEN protein is present (25). Ishikawa and AN3CA cells also exhibit mutations in the PI3K subunit PIKR1, whereas HEC1A cells exhibit mutations in the PI3K subunit PIK3CA or KRAS (25).

In all three EC cell lines restoration of miR-29c reduced ITGB1 and MDM2 expression levels. ITGB1 is involved in cell adhesion, metastatic diffusion, cell repair and tumour growth (23). ITGB1 is a confirmed target of miR-29c in gastric cancer and their expression is correlated with clinical outcome (29). ITGB1 has been shown to promote the invasive capacity of endometrial stromal cells in a previous study investigating endometriosis (30). MDM2 is an oncogene that promotes tumour occurrence and development predominantly by inhibiting the p53 tumour suppressor activity (24). Jiang et al (31) found increased MDM2 mRNA and protein levels with increasing EC grade.

Numerous genes associated with cell proliferation were found to be regulated by miR-29c; however, the genes regulated by miR-29c in each cell line suggested that the mechanism by which cell proliferation is regulated may be different among cell lines.

In Ishikawa and HEC1A cells, miR-29c overexpression reduced the expression of genes associated with cell proliferation. HBP1 was downregulated by miR-29c in both Ishikawa and HEC1A cells. HBP1 is a transcriptional repressor and is associated with the induction of cyclins involved in cellular proliferation (32). HBP1 knockdown arrests cells in $\mathrm{G}_{0} /$ phase, decreases those in $\mathrm{S}$ phase, but does not affect apoptosis in neural progenitor cells (33).

SGK1 is a serine/threonine kinase that modulates cancer cell growth and survival (34). SGK1 expression was reduced following miR-29c overexpression in Ishikawa cells; however, these cells did not exhibit significant changes in proliferation. SGK1 is increased in EC compared with benign endometrial tissue, and its inhibition via the small molecule inhibitor SI113 results in decreased cell viability and increased apoptosis and autophagy (34).

SIRT1 is involved in cell proliferation, apoptosis and metastasis (35). miR-29c overexpression significantly reduced SIRT1 in HEC1A cells. There are conflicting results published on the expression of SIRT1 in EC $(36,37)$. Functionally, SIRT1 enhances EC growth, progression and chemo-resistance in EC cell lines in vitro and in vivo (37). SIRT1 is a confirmed target of miR-29c and its overexpression recapitulates SIRT1 knockdown and suppresses hepatocellular carcinoma cell growth (38).

VEGFA is upregulated in many cancer types including $\mathrm{EC}$, and VEGFA upregulation is associated with proliferation, migration, differentiation, blood vessel formation and poor prognosis (39). miR-29c overexpression reduced VEGFA production in HEC1A cells. VEGFA is increased in EC, and may play a role in angiogenesis during tumour growth, and it has been identified as a target of miR-29b (11).

In AN3CA cells, miR-29c reduced the expression levels of cell cycle and anti-apoptotic proteins, thus influencing these processes, as assessed by flow cytometry. CDC42 is a confirmed target of miR-29c (40) and plays a role in cell cycle regulation, cell-cell adhesion, migration and cancer progression (41). Following miR-29c overexpression, the resulting loss of CDC42 in the AN3CA cells coincides with altered $G_{0} / G_{1}$ and $\mathrm{G}_{2} / \mathrm{M}$ phase cell populations for which CDC42 is known to be required (42). In cervical cancer, CDC42 overexpression promoted migration via the formation of cellular filopodia $(42,43)$, and overexpression of the miR-29a/b/c family was able to reduce migration and invasion of glioma cell lines (U87MG and U251) via the reduction of CDC42 (44). Other studies identified a correlation between increased CDC42 levels and higher cancer stage $(42,45,46)$.

MCL1 is an antiapoptotic protein. MCL1 is a direct target of miR-29c, and miR-29c overexpression is able to 
knockdown the expression of MCL1 in lymphoma (15). Immunohistochemistry analysis of high-grade EC tumour samples identified that MCL1 is overexpressed compared with benign cells (47). In the present study, a significant reduction in MCL1 expression in Ishikawa and AN3CA cell lines following miR-29c overexpression was found, and this reduction was associated with the increased apoptotic rate seen in the AN3CA cell line.

A number of factors identified in the results of the present study, including ITGB1, CDC42, SIRT1 and VEGFA, can regulate EC cell adhesion, migration and invasion; however, these functions were not directly investigated in the present study. The role of the other miR-29 family members (a and b) in regulating EC should also be considered. Similarly to miR-29c, miR-29a regulates EC cell proliferation and apoptosis (13) via TPX2 microtubule nucleation factor, a gene which was not investigated in the present study. Importantly, the therapeutic potential of each miR-29 family member and their specific roles in EC requires further investigation.

In the present study, the endogenous levels of miR-29c in HEC1A cells were not reduced, in contrast with the Ishikawa and AN3CA cells. The expression level of miR-29c in HEC1A was similar to the levels found in the non-cancerous endometrial epithelial cells. Downregulation of miR-29c, identified in EC, increased HEC1A proliferation and COL4A1 expression, but had no effect on cell cycle. To the best of our knowledge, the present study is the third study to identify COL4A1 as a target gene of factors dysregulated in EC; in fact, COL4A1 production is regulated by Kruppel-like factor 9 in HEC1A cells (48) and Galectin-7 in Ishikawa cells (49). Collagen IV is the most abundant structural component of cellular basement membranes, involved in defining compartments that separate endothelial and epithelial layers from the underlying mesenchyme (50). Collagen IV is necessary for cell adhesion and acts by binding to cell surface receptors, mainly integrin $\beta 1$ (50). Within the endometrium, collagen IV abundance changes across the menstrual cycle and within endometrial compartments (51), and the loss of collagen IV within the epithelial compartments is associated with primary infertility (16). The direct role of COL4A1 in EC remains to be elucidated.

In conclusion, miR-29c was downregulated in EC, possibly resulting in increased proliferation and COL4A1 production. Following miR-29c overexpression in EC cell lines, a reduction in proliferation and/or cell cycle progression were identified in HEC1A and AN3CA cells. miR-29c target genes involved in adhesion, migration, angiogenesis, proliferation and apoptosis were also downregulated following miR-29c overexpression, suggesting that miR-29c may be a potential therapeutic target for EC. Finally, the precise mechanism by which miR-29c acts is likely not identical among the three EC cell lines investigated, highlighting the importance of molecular characterization of tumours. The present study provided novel insights into specific treatments for different disease stages of EC.

\section{Acknowledgements}

The authors would like to thank Dr Masashi Takamura, University Hospital of Tokyo (Tokyo, Japan), for primary cell culture preparations and Ms. Katarzyna Rainczuk, Hudson
Institute of Medical Research (Clayton, Australia), for technical assistance. These data were presented at the Australian ESA-SRB meeting in Adelaide, 19-22 August 2018 (abstract no. 61).

\section{Funding}

The present study was supported by the Victorian Government's Operational Infrastructure Support Program. ED was supported by The National Health and Medical Research Council (Australia) Senior Research Fellowship (grant no. 550905).

\section{Availability of data and materials}

The datasets used and/or analyzed during the current study are available from the corresponding author on reasonable request.

\section{Authors' contributions}

MVS and ED conceived and designed the experiments, critically analysed the data and reviewed the manuscript. MVS wrote the manuscript, and contributed to data collection and analysis. EM analysed the data and wrote the manuscript. MG contributed to data collection and analysis and manuscript review. KN contributed to data analysis. All authors read and approved the final manuscript.

\section{Ethics approval and consent to participate}

The present study was approved by the Monash Health Human Research and Ethics Committee (approval no. 06014C) and the Victorian Cancer Biobank (project no. 13018). Informed consent was obtained from each patient.

\section{Patient consent for publication}

Participant consent for the publication of research generated from anonymous samples was obtained.

\section{Competing interests}

The authors declare that they have no competing interests.

\section{References}

1. Ferlay J, Shin HR, Bray F, Forman D, Mathers C and Parkin DM: Estimates of worldwide burden of cancer in 2008: GLOBOCAN 2008. Int J Cancer 127: 2893-2917, 2010.

2. Soliman PT, Oh JC, Schmeler KM, Sun CC, Slomovitz BM, Gershenson DM, Burke TW and Lu KH: Risk factors for young premenopausal women with endometrial cancer. Obstet Gynecol 105: 575-580, 2005.

3. Bilyk O, Coatham M, Jewer $M$ and Postovit LM: Epithelial-to-Mesenchymal Transition in the female reproductive tract: From normal function to disease pathology. Front Oncol 7: 145,2017

4. Hong B, Le Gallo M and Bell DW: The mutational landscape of endometrial cancer. Curr Opin Genet Dev 30: 25-31, 2015.

5. Di Cristofano A and Ellenson LH: Endometrial carcinoma. Annu Rev Pathol 2: 57-85, 2007.

6. Piulats JM, Guerra E, Gil-Martin M, Roman-Canal B, Gatius S, Sanz-Pamplona R, Velasco A, Vidal A and Matias-Guiu X: Molecular approaches for classifying endometrial carcinoma. Gynecol Oncol 145: 200-207, 2017. 
7. Arend RC, Jones BA, Martinez A and Goodfellow P: Endometrial cancer: Molecular markers and management of advanced stage disease. Gynecol Oncol 150: 569-580, 2018.

8. Kim VN: MicroRNA biogenesis: Coordinated cropping and dicing. Nat Rev Mol Cell Biol 6: 376-385, 2005.

9. Schmitt MJ, Margue C, Behrmann I and Kreis S: MiRNA-29: A microRNA family with tumor-suppressing and immune-modulating properties. Curr Mol Med 13: 572-585, 2013.

10. Castilla MÁ, Moreno-Bueno G, Romero-Pérez L, Van De Vijver K, Biscuola M, López-García MÁ, Prat J, Matías-Guiu X, Cano A, Oliva E and Palacios J: Micro-RNA signature of the epithelial-mesenchymal transition in endometrial carcinosarcoma. J Pathol 223: 72-80, 2011.

11. Chen HX, Xu XX, Tan BZ, Zhang Z and Zhou XD: Micro-RNA-29b inhibits angiogenesis by targeting VEGFA through the MAPK/ERK and PI3K/Akt signaling pathways in endometrial carcinoma. Cell Physiol Biochem 41: 933-946, 2017.

12. Hiroki E, Akahira J, Suzuki F, Nagase S, Ito K, Suzuki T, Sasano $\mathrm{H}$ and Yaegashi N: Changes in microRNA expression levels correlate with clinicopathological features and prognoses in endometrial serous adenocarcinomas. Cancer Sci 101: 241-249, 2010.

13. Jiang T, Sui D, You D, Yao S, Zhang L, Wang Y, Zhao J and Zhang Y: miR-29a-5p inhibits proliferation and invasion and induces apoptosis in endometrial carcinoma via targeting TPX2. Cell Cycle 17: 1268-1278, 2018.

14. Ushakov DS, Dorozhkova AS, Babayants EV, Ovchinnikov VY, Kushlinskii DN, Adamyan LV, Gulyaeva LF and Kushlinskii NE: Expression of microRNA potentially regulated by AhR and CAR in malignant tumors of the endometrium. Bull Exp Biol Med 165: 688-691, 2018

15. Mazzoccoli L, Robaina MC, Apa AG, Bonamino M, Pinto LW, Queiroga E, Bacchi CE and Klumb CE: MiR-29 silencing modulates the expression of target genes related to proliferation, apoptosis and methylation in Burkitt lymphoma cells. J Cancer Res Clin Oncol 144: 483-497, 2018.

16. Griffiths M, Van Sinderen M, Rainczuk K and Dimitriadis E: miR-29c overexpression and COL4A1 downregulation in infertile human endometrium reduces endometrial epithelial cell adhesive capacity in vitro implying roles in receptivity. Sci Rep 9: 8644, 2019.

17. Long M, Wan $X$, La $X$, Gong $X$ and Cai $X$ : miR-29c is downregulated in the ectopic endometrium and exerts its effects on endometrial cell proliferation, apoptosis and invasion by targeting c-Jun. Int J Mol Med 35: 1119-1125, 2015.

18. Marwood M, Visser K, Salamonsen LA and Dimitriadis E: Interleukin-11 and leukemia inhibitory factor regulate the adhesion of endometrial epithelial cells: Implications in fertility regulation. Endocrinology 150: 2915-2923, 2009.

19. Winship A, Van Sinderen M, Rainczuk K and Dimitriadis E Therapeutically blocking Interleukin-11 Receptor-alpha enhances doxorubicin cytotoxicity in high grade type I endometrioid tumours. Oncotarget 8: 22716-22729, 2017.

20. Van Sinderen M, Cuman C, Winship A, Menkhorst E and Dimitriadis E: The chrondroitin sulfate proteoglycan (CSPG4) regulates human trophoblast function. Placenta 34: 907-912, 2013.

21. Livak K and Schmittgen T: Analysis of relative gene expression data using real-time quantitative PCR and the 2(-Delta DeltaC(T)) method. Methods 25: 402-408, 2001

22. Licholai S, Szczekilk W and Sanak M: miR-29c-3p is an effective biomarker of abdominal aortic aneurysm in patients undergoing elective surgery. MicroRNA 5: 124-131, 2016.

23. Xu Z, Zou L, Gang M, Wu X, Huang F, Feng T, Li S, Lin Q, He X, Liu $\mathrm{Z}$ and Cao $\mathrm{X}$ : Integrin b1 is a critical effector in promoting metastasis and chemo-resistance of esophageal squamous cell carcinoma. Am J Cancer Res 7: 531-542, 2017.

24. Gupta A, Shah K, Oza MJ and Behl T: Reactivation of p53 gene by MDM2 inhibitors: A novel therapy for cancer treatment Biomed Pharmacother 109: 484-492, 2019.

25. Weigelt B, Warne PH, Lambros MB, Reis-Filho JS and Downward J: PI3K pathway dependencies in endometrial cancer cell lines. Clin Cancer Res 19: 3533-3544, 2013.

26. Bhardwaj A, Singh H, Rajapakshe K, Tachibana K, Ganesan N, Pan Y, Gunaratne PH, Coarfa C and Bedrosian I: Regulation of miRNA-29c and its downstream pathways in preneoplastic progression of triple-negative breast cancer. Oncotarget 8: 19645-19660, 2017.
27. Chen G, Zhou T, Li Y, Yu Z and Sun L: p53 target miR-29c-3p suppresses colon cancer cell invasion and migration through inhibition of PHLDB2. Biochem Biophys Res Commun 487: 90-95, 2017

28. Chuang TD and Khorram O: Mechanisms underlying aberrant expression of miR-29c in uterine leiomyoma. Fertil Steril 105: 236-245 e1, 2016.

29. He B, Xiao YF, Tang B, Wu YY, Hu CJ, Xie R, Yang X, Yu ST, Dong H, Zhao XY, et al: hTERT mediates gastric cancer metastasis partially through the indirect targeting of ITGB1 by microRNA-29a. Sci Rep 6: 21955, 2016

30. Chen J, Gu L, Ni J, Hu P, Hu K and Shi YL: MiR-183 Regulates ITGB1P Expression and Promotes Invasion of Endometrial Stromal Cells. Biomed Res Int 2015: 340218, 2015.

31. Jiang Z, Xu W, Dan G, Liu Y and Xiong J: P53 and murine double mimute 2 (MDM2) expression changes and significance in different types of endometrial lesions. Med Sci Monit 22: 4786-4793, 2016.

32. Bollaert E, de Rocca Serra A and Demoulin JB: The HMG box transcription factor HBP1: A cell cycle inhibitor at the crossroads of cancer signaling pathways. Cell Mol Life Sci 76: 1529-1539, 2019.

33. He S, Yang S, Niu M, Zhong Y, Dan Gao, Zhang Y, Ma H, Xiong W, Zhou M, Zhou Y, et al: HMG-box transcription factor 1: A positive regulator of the G1/S transition through the Cyclin-CDK-CDKI molecular network in nasopharyngeal carcinoma. Cell Death Dis 9: 100, 2018.

34. Conza D, Mirra P, Cali G, Tortora T, Insabato L, Fiory F, Schenone S, Amato R, Beguinot F, Perrotti N and Ulianich L: The SGK1 inhibitor SI113 induces autophagy, apoptosis, and endoplasmic reticulum stress in endometrial cancer cells. J Cell Physiol 232: 3735-3743, 2017.

35. Palmirotta R, Cives M, Della-Morte D, Capuani B, Lauro D, Guadagni F and Silvestris F: Sirtuins and cancer: Role in the epithelial-mesenchymal transition. Oxid Med Cell Longev 2016: 3031459, 2016.

36. Bartosch C, Monteiro-Reis S, Almeida-Rios D, Vieira R, Castro A, Moutinho M, Rodrigues M, Graça I, Lopes JM and Jerónimo C: Assessing sirtuin expression in endometrial carcinoma and non-neoplastic endometrium. Oncotarget 7: 1144-1154, 2016.

37. Asaka R, Miyamoto T, Yamada Y, Ando H, Mvunta DH, Kobara $\mathrm{H}$ and Shiozawa T: Sirtuin 1 promotes the growth and cisplatin resistance of endometrial carcinoma cells: A novel therapeutic target. Lab Invest 95: 1363-1373, 2015.

38. Bae HJ, Noh JH, Kim JK, Eun JW, Jung KH, Kim MG, Chang YG, Shen Q, Kim SJ, Park WS, et al: MicroRNA-29c functions as a tumor suppressor by direct targeting oncogenic SIRT1 in hepatocellular carcinoma. Oncogene 33: 2557-2567, 2014.

39. Mahecha AM and Wang $\mathrm{H}$ : The influence of vascular endothelial growth factor-A and matrix metalloproteinase- 2 and -9 in angiogenesis, metastasis, and prognosis of endometrial cancer. Onco Targets Ther 10: 4617-4624, 2017.

40. Park SY, Lee JH, Ha M, Nam JW and Kim VN: miR-29 miRNAs activate $\mathrm{p} 53$ by targeting p 85 alpha and CDC42. Nat Struct Mol Biol 16: 23-29, 2009

41. Qadir MI, Parveen A and Ali M: Cdc42: Role in cancer management. Chem Biol Drug Des 86: 432-439, 2015.

42. Ye H, Zhang Y, Geng L and Li Z: Cdc42 expression in cervical cancer and its effects on cervical tumor invasion and migration. Int J Oncol 46: 757-763, 2015.

43. Feng Y, Fang S and Li M: Expression of P21-activated kinase 1 and cell division control protein 42 homolog correlates with clinicopathological features and prognosis in cervical carcinoma. J Obstet Gynaecol Res 42: 860-869, 2016.

44. Shi C, Ren L, Sun C, Yu L, Bian X, Zhou X, Wen Y, Hua D, Zhao S, Luo W, et al: miR-29a/b/c function as invasion suppressors for gliomas by targeting CDC42 and predict the prognosis of patients. Br J Cancer 117: 1036-1047, 2017.

45. Yang D, Zhang Y, Cheng Y, Hong L, Wang C, Wei Z, Cai Q and Yan R: High expression of cell division cycle 42 promotes pancratic cancer growth and predicts poor outcome of pancreatic cancer patients. Dig Dis Sci 62: 958-967, 2017.

46. Kamai T, Yamanishi T, Shirataki H, Takagi K, Asami H, Ito Y and Yoshida K: Overexpression of RhoA, Rac1 and Cdc42 GTPases is associated with progression in testicular cancer. Clin Cancer Res 10: 4799-4805, 2004. 
47. Konno Y, Dong P, Xiong Y, Suzuki F, Lu J, Cai M, Watari H, Mitamura T, Hosaka M, Hanley SJ, et al: MicroRNA-101 targets EZH2, MCL-1 and FOS to suppress proliferation, invasion and stem cell-like phenotype of aggressive endometrial cancer cells. Oncotarget 5: 6049-6062, 2014.

48. Simmen F, Su Y, Xiao R, Zeng Z and Simmen RC: The Kruppel-like factor 9 (KLF9) network in HEC-1-A endometrial carcinoma cells suggests the carcinogenic potential of dys-regulated KLF9 expression. Reprod Biol Endocrinol 6: 41, 2008
49. Menkhorst E, Griffiths M, Van Sinderen M, Rainczuk K, Niven K and Dimitriadis E: Galectin-7 is elevated in endometrioid (type I) endometrial cancer and promotes cell migration. Oncol Lett 16: 4721-4728, 2018.

50. Van Agtmael T and Bruckner-Tuderman L: Basement membranes and human disease. Cell Tissue Res 339: 167-188, 2010.

51. Kelly FD, Tawia SA and Rogers PA: Immunohistochemical characterization of human endometrial microvascular basement membrane components during the normal menstrual cycle. Hum Reprod 10: 268-276, 1995. 